\title{
Suicidal Ideation and Suicide Attempts in Middle-Aged Women Attending a Primary Care Center: A Cross-Sectional Study in Mexico
}

\author{
Cosme Alvarado-Esquivel
}

\begin{abstract}
Background: Very little is known about suicide attempts in middleaged women in Mexico. The aims of this study were to determine: 1) the prevalence of suicidal ideation and suicide attempts in middleaged women in Durango City, Mexico; and 2) the suicide attempt prevalence association with the sociodemographic, clinical, and behavioral characteristics of the women studied.
\end{abstract}

Methods: Through a cross-sectional study, 395 middle-aged women were surveyed. Suicidal ideation, suicide attempt data, and the characteristics of the women were obtained with the aid of a questionnaire. Bivariate and multivariate analyses were used to determine the association between suicide attempts and the characteristics of women.

Results: Of the 395 women (mean age: $46.97 \pm 5.34$ years) studied, $50(12.7 \%)$ had suicide ideation, and $20(5.1 \%)$ had suicide attempts. The number of suicide attempts among these 20 women varied from 1 to 10 times. The most frequent method used for suicide attempt was intake of medicaments (in 11 women). None of the sociodemographic characteristics was associated with suicide attempts. Women with suicide ideation, abdominal pain frequently, and reflexes impairment had a significantly higher frequency of suicide attempts than those without these characteristics. Logistic regression of behavioral variables showed that only the variable alcohol consumption was independently associated with suicide attempts (odds ratio $=2.82 ; 95 \%$ confidence interval: $1.01-7.84 ; \mathrm{P}=0.04$ ).

Conclusions: Results suggest that suicidal ideation and suicide attempts are prevalent among women of middle-age attending a public primary care center in Durango City. This is the first report of an association between suicide attempts and reflexes impairment. Factors associated with suicide attempts found in this study may help in the design of preventive measures against suicide.

Keywords: Suicidal ideation; Suicide attempts; Prevalence; Women;

Manuscript submitted May 31, 2018, accepted June 28, 2018

Faculty of Medicine and Nutrition, Juarez University of Durango State, Avenida Universidad S/N, 34000 Durango, Dgo, Mexico.

Email: alvaradocosme@yahoo.com

doi: https://doi.org/10.14740/jocmr3501w
Cross-sectional study

\section{Introduction}

Suicide is a serious public health concern worldwide [1]. Women commit fewer suicides than men but make more frequent attempts $[2,3]$. In two nationally representative surveys in the USA, most adults with recent suicide attempts were females and younger than 50 years [4]. The incidence of parasuicide or attempted suicide is 10 - 20 times higher than that of completed suicide [5]. Suicide appears to be closely related to psychiatric morbidity [6]. In general population, almost $1 \%$ of people attempt suicide. In a study of data of two national surveys of alcohol and related conditions in the USA, the frequency of adults making a recent suicide attempt increased from $0.62 \%$ in the years $2004-2005$ to $0.79 \%$ in the years $2012-2013$ [4]. In a meta-analysis of eight studies about the prevalence of suicide attempts in the general population of mainland China, researchers found a $0.8 \%$ pooled lifetime prevalence of suicide attempts [7]. On the other hand, more than half of suicide victims contact their general practitioners in the month before the commitment of suicide $[8,9]$. In outpatients of primary care settings, the prevalence of suicide attempts varies among countries, i.e., 1.2\% in Morocco [10], and 6.9\% in India [11].

Very little is known about the epidemiology of suicide attempts in women in Mexico. To the best of my knowledge, the epidemiology of suicide attempt in middle-aged women in Mexico has not been studied. This study was aimed to determine: 1) the prevalences of suicide ideation and attempts in middle-aged women attending a primary health care center in Durango City, Mexico; and 2) the suicide attempt prevalence association with the sociodemographic, clinical, and behavioral characteristics of the women studied.

\section{Materials and Methods}

\section{Study design and women surveyed}

A cross-sectional study of 395 middle-aged women was performed. Participants were enrolled in the study in a primary care center in the northern Mexican city of Durango. Inclu- 
sion criteria were: 1) women aged 38 - 56 years; 2) attended in the Health Care Center \#2 of the Secretary of Health in Durango City; and 3) who voluntarily accepted to participate in the study.

\section{Sociodemographic, clinical, and behavioral data of partici- pants}

Sociodemographic, clinical, and behavioral characteristics of the women studied were recorded in a questionnaire during face-to-face interviews. Sociodemographic items included age, birthplace, educational status, residence place, occupation, and socio-economic status. Clinical data included history of suicidal ideation, and suicide attempts (number and method of suicide attempts), presence of any underlying disease, obstetric history (pregnancies, deliveries, miscarriages, and cesarean sections), frequent abdominal pain or headache, impairments in memory, reflexes, hearing and vision, and history of surgery or blood transfusion. Suicidal ideation and suicide attempts were diagnosed according to definitions of the Centers for Disease Control and Prevention (https://www.cdc. gov/violenceprevention/suicide/definitions.html). Behavioral data of women included traveling (national and international trips), consumption of alcohol, smoking, drug abuse, sexual promiscuity, type of sexuality (heterosexual, homosexual, and bisexual), crowding at home, and contact with animals (dogs, cats, birds, and farm animals). Alcohol consumption was considered when a participant used to drink any quantity of alcohol to socialize, celebrate, or relax regardless the frequency of consumption. Drug abuse was considered when a participant consumed any illegal drug regardless the quantity or frequency of consumption.

\section{Statistical analysis}

The statistical analysis was performed using the software Epi Info 7, and SPSS 15.0 (SPSS Inc. Chicago, IL). The sample size was calculated using the following data: 1) a reference seroprevalence of $9.2 \%$ [11] as the expected frequency of suicide attempts in women, 2) a population size of 50,000, 3) a $5 \%$ of confidence limits, and 4) a $99.9 \%$ confidence level. The result of the sample size calculation was 359 subjects. The association between the presence of suicide attempts and the sociodemographic, clinical, and behavioral characteristics of participants was assessed with the Pearson's Chi-squared test or the two-tailed Fisher's exact test (when values were small). Characteristics with a $\mathrm{P}$ value equal to or less than 0.05 obtained in the bivariate analysis were selected for multivariate analysis. Odds ratio (OR) and 95\% confidence interval (CI) were calculated by logistic regression using the Enter method. Statistical significance was set at a $\mathrm{P}$ value $<0.05$.

\section{Ethics aspects}

The Ethics Committee of the General Hospital of the Secretary of Health in Durango City, Mexico approved this project. Participation in the study was voluntary. All participants provided a written informed consent.

\section{Results}

Of the 395 women (mean age: $46.97 \pm 5.34$ years; range 38 - 56 years) studied, 50 (12.7\%) had suicidal ideation, and 20 $(5.1 \%)$ had suicide attempts. The number of suicide attempts among these 20 women varied from 1 to 10 times: 11 women had attempted once, six women between 2 and 5 times, one woman between 6 and 10 times, and two women did not provide the number of suicide attempts. Four women had attempted suicide during the last 12 months before the interview, 15 women attempted suicide more than 12 months ago before the interview, and one woman did not provide the date she attempted suicide. The most frequent method used for suicide attempt was intake of medicaments (in 11 women), followed by hanging up (in four women), cuts (in three women), and a shot with a weapon (in one woman). Two women did not provide the method used for suicide attempt. A correlation of the sociodemographic data and the prevalence of suicide attempts in the women studied are shown in Table 1. Bivariate analysis of the sociodemographic characteristics showed that none of these variables had $\mathrm{P}$ values $\leq 0.05$. Whereas bivariate analysis of clinical data showed that women with suicidal ideation, abdominal pain frequently, and reflexes impairment had a significantly higher frequency of suicide attempts than those without these characteristics. Table 2 shows a correlation between suicide attempts and clinical characteristics. Bivariate analysis of the behavioral data showed five variables with a $\mathrm{P}$ value $\leq 0.05$ : national trips, alcohol consumption, tobacco consumption, drug abuse, and crowding at home. Table 3 shows a correlation of the behavioral data and prevalence of suicide attempts. Further analysis by logistic regression of behavioral variables with $\mathrm{P} \leq 0.05$ obtained by bivariate analysis showed that only the variable alcohol consumption was associated with suicide attempts $(\mathrm{OR}=2.82 ; 95 \% \mathrm{CI}$ : $1.01-7.84 ; \mathrm{P}=$ 0.04) (Table 4).

\section{Discussion}

The epidemiology of suicide attempts in middle-aged women in primary care settings in Mexico is largely unknown. Therefore, this study was aimed to determine the prevalences of suicidal ideation and suicide attempts in middle-aged women attending a primary health care center in the northern Mexican city of Durango; and the association between suicide attempt prevalence and the sociodemographic, clinical, and behavioral characteristics of the women studied. Prevalences of $12.7 \%$ and $5.1 \%$ for suicidal ideation and suicide attempts were found, respectively. There is scanty information regarding suicidal ideation and suicide attempts in people attending primary care settings to compare with the results of the present work. In a study of patients in four primary care clinics in Lithuania, researchers found that $6 \%$ of patients reported suicidal idea- 
Table 1. Correlation of Socio-Demographic Characteristics of Women and Prevalence of Suicide Attempts

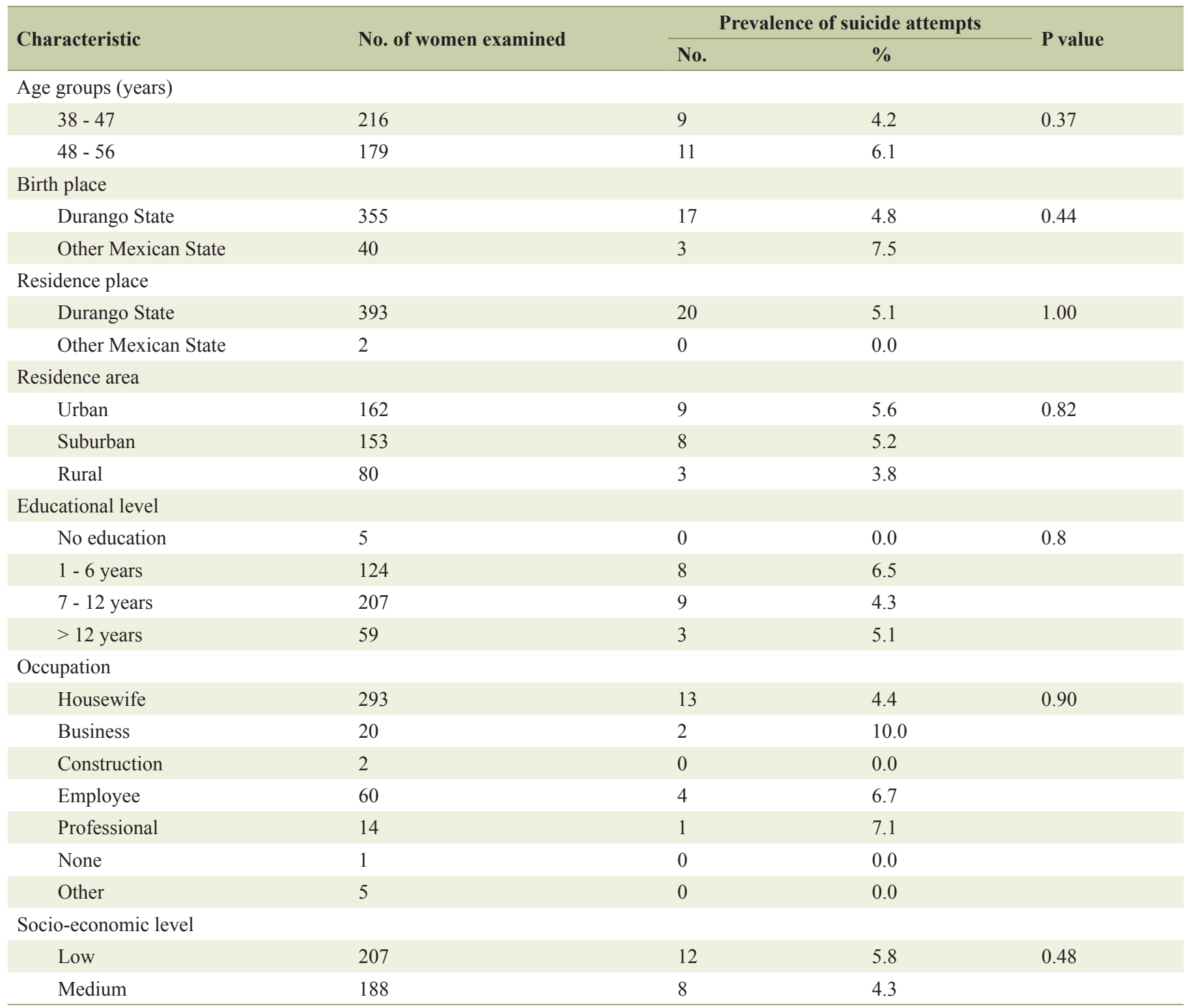

tion [12]. In a study of African-American women in a community-based primary health care center, researchers found a $10 \%$ prevalence of a history of suicidal ideation [13]. The $12.7 \%$ prevalence of suicidal ideation found in the present study is thus higher than the prevalences found in women in primary care clinics in Lithuania [12], and in African-American women in the USA [13]. The high prevalence of suicidal ideation in middle-aged women found in the present work agrees with the high prevalence of suicidal ideation found in women aged 44 - 50 years found in a national study about trends of suicidal ideation in England [14]. Concerning the 5.1\% prevalence of suicide attempts found in the present study, it is lower than the $9.2 \%$ prevalence reported in women in six primary care settings in India [11]. Despite suicidal behavior is prevalent in patients in primary care clinics, and that most suicidal individ- uals had contacted general practitioners or other primary care services during the previous 12 months before the suicide attempts $[8,9,15]$, patients planning suicide attempts attending these clinics are still poorly recognized, and the epidemiology of suicide attempts in these patients in primary care has been poorly studied.

In the present study, bivariate analysis showed three clinical characteristics associated with suicide attempts: suicide ideation, abdominal pain frequently, and reflexes impairment. It is unclear why suicide attempters had significantly higher frequencies of abdominal pain and reflexes impairment than no attempters. Intriguingly, in a German study, recurrent pain in any of three areas of pain assessed (general pain, abdominal pain, and headage) was significantly associated with suicidal ideation and suicide attempts in adolescents [16]. In addition, 
Table 2. Bivariate Analysis of Clinical Data and Suicide Attempts in the Women Studied

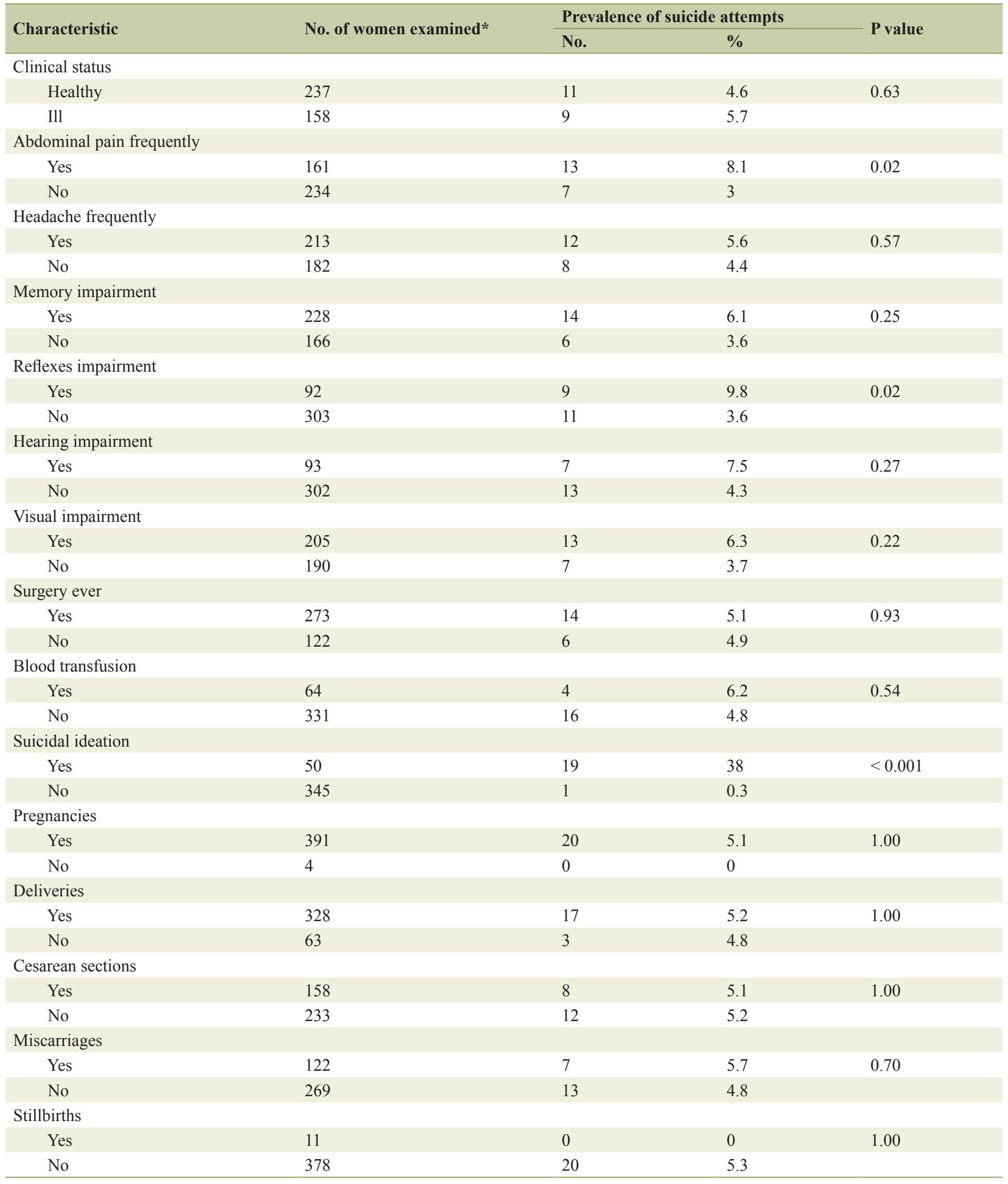

*Sums may not add up to 395 because of some missing values. 
Table 3. Bivariate Analysis of Behavioral Characteristics and Prevalence of Suicide Attempts in the Women Studied

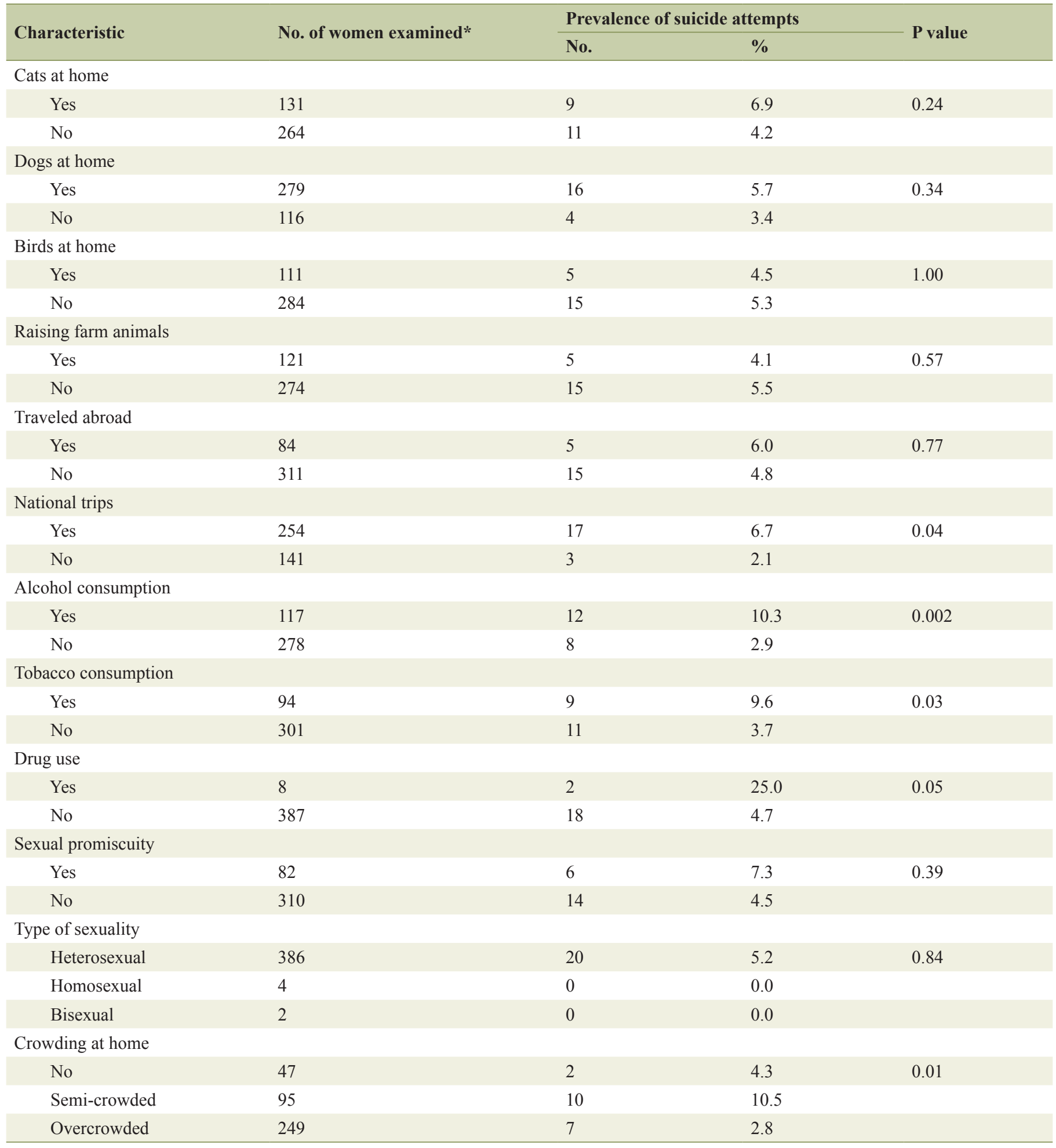

*Sums may not add up to 395 because of some missing values.

elevated suicide risk has been associated with ambiguous diagnoses (psychogenic pain and abdominal pain) [17]. To the best of my knowledge, the association between suicide attempts and reflexes impairment has not been previously reported. It is possible that reflexes impairment might suggest poor quality of live or depression that could lead to suicide attempts. Fur- 
Table 4. Multivariate Analysis of Selected Behavioral Characteristics of Women and Their Association With Suicide Attempts

\begin{tabular}{llll}
\hline Characteristic & Odds ratio & $\mathbf{9 5 \%}$ confidence interval & P value \\
\hline National trips & 3.88 & $0.85-17.72$ & 0.07 \\
Alcohol consumption & 2.82 & $1.01-7.84$ & 0.04 \\
Tobacco consumption & 1.76 & $0.62-4.95$ & 0.28 \\
Drug abuse & 5.25 & $0.80-34.36$ & 0.08 \\
Crowding at home & 1.49 & $0.80-2.78$ & 0.2 \\
\hline
\end{tabular}

ther studies to confirm this association are needed.

Of the behavioral characteristics assessed in the present study, only the variable alcohol consumption was associated with suicide attempts. Alcohol consumption has been associated with suicide attempts in several studies. For instance, this association was found in adolescents in Mexico [18], and Chile [19], women in Sri Lanka [20], and pregnant women with mental disorders in France [21].

The present study has limitations: firstly, most women studied resided in urban and suburban areas and few resided in rural Durango; and secondly, women were enrolled in a health center of a health institution in Durango City, but there are more health centers in two more health institutions in the same city. Therefore, further studies in rural area and more health institutions to determine the epidemiology of suicide attempts in middle-aged women in Durango, Mexico should be conducted.

\section{Conclusions}

Results suggest that suicidal ideation and suicide attempts are prevalent among women of middle-age attending a public primary care center in Durango City. This is the first report of an association between suicide attempts and reflexes impairment. Factors associated with suicide attempts found in this study may help in the design of preventive measures against suicide.

\section{Conflict of Interest}

None.

\section{Grant Support}

None.

\section{References}

1. Choi SB, Lee W, Yoon JH, Won JU, Kim DW. Risk factors of suicide attempt among people with suicidal ideation in South Korea: a cross-sectional study. BMC Public Health. 2017;17(1):579.

2. Canetto SS. Women and suicidal behavior: a cultural analysis. Am J Orthopsychiatry. 2008;78(2):259-266.

3. Mendez-Bustos P, Lopez-Castroman J, Baca-Garcia E, Ceverino A. Life cycle and suicidal behavior among women. ScientificWorldJournal. 2013;2013:485851.

4. Olfson M, Blanco C, Wall M, Liu SM, Saha TD, Pickering RP, Grant BF. National trends in suicide attempts among adults in the United States. JAMA Psychiatry. 2017;74(11):1095-1103.

5. Diekstra RF, Gulbinat W. The epidemiology of suicidal behaviour: a review of three continents. World Health Stat Q. 1993;46(1):52-68.

6. Lejoyeux M, Leon E, Rouillon F. [Prevalence and risk factors of suicide and attempted suicide]. Encephale. 1994;20(5):495-503.

7. Cao XL, Zhong BL, Xiang YT, Ungvari GS, Lai KY, Chiu HF, Caine ED. Prevalence of suicidal ideation and suicide attempts in the general population of China: A meta-analysis. Int J Psychiatry Med. 2015;49(4):296-308.

8. McDowell AK, Lineberry TW, Bostwick JM. Practical suicide-risk management for the busy primary care physician. Mayo Clin Proc. 2011;86(8):792-800.

9. Rihmer Z, Dome P, Gonda X. The role of general practitioners in prevention of depression-related suicides. Neuropsychopharmacol Hung. 2012;14(4):245-251.

10. Oneib B, Sabir M, Otheman Y, Abda N, Ouanass A. Suicidal ideations, plans and attempts in primary care: crosssectional study of consultants at primary health care system in Morocco. Pan Afr Med J. 2016;24:274.

11. Indu PS, Anilkumar TV, Pisharody R, Russell PSS, Raju D, Sarma PS, Remadevi S, et al. Prevalence of depression and past suicide attempt in primary care. Asian J Psychiatr. 2017;27:48-52.

12. Bunevicius R, Liaugaudaite V, Peceliuniene J, Raskauskiene N, Bunevicius A, Mickuviene N. Factors affecting the presence of depression, anxiety disorders, and suicidal ideation in patients attending primary health care service in Lithuania. Scand J Prim Health Care. 2014;32(1):2429.

13. Holden KB, Bradford LD, Hall SP, Belton AS. Prevalence and correlates of depressive symptoms and resiliency among African American women in a community-based primary health care center. J Health Care Poor Underserved. 2013;24(4 Suppl):79-93.

14. Spiers N, Bebbington PE, Dennis MS, Brugha TS, McManus S, Jenkins R, Meltzer H. Trends in suicidal ideation in England: the national psychiatric morbidity surveys of 2000 and 2007. Psychol Med. 2014;44(1):175183. 
15. Hintikka J, Viinamaki H, Tanskanen A, Kontula O, Koskela K. Suicidal ideation and parasuicide in the Finnish general population. Acta Psychiatr Scand. 1998;98(1):2327.

16. Koenig J, Oelkers-Ax R, Parzer P, Haffner J, Brunner R, Resch F, Kaess M. The association of self-injurious behaviour and suicide attempts with recurrent idiopathic pain in adolescents: evidence from a population-based study. Child Adolesc Psychiatry Ment Health. 2015;9:32.

17. Newton-John TR. Negotiating the maze: risk factors for suicidal behavior in chronic pain patients. Curr Pain Headache Rep. 2014;18(9):447.

18. Valdez-Santiago R, Solorzano EH, Iniguez MM, Burgos LA, Gomez Hernandez H, Martinez Gonzalez A. Attempted suicide among adolescents in Mexico: preva- lence and associated factors at the national level. Inj Prev. 2017.

19. Salvo GL, Castro SA. [Association of loneliness, impulsivity and alcohol use with suicidal behavior in adolescents]. Rev Med Chil. 2013;141(4):428-434.

20. Knipe DW, Gunnell D, Pearson M, Jayamanne S, Pieris $\mathrm{R}$, Priyadarshana C, Weerasinghe M, et al. Attempted suicide in Sri Lanka - An epidemiological study of household and community factors. J Affect Disord. 2018;232:177184.

21. Gressier F, Guillard V, Cazas O, Falissard B, GlangeaudFreudenthal NM, Sutter-Dallay AL. Risk factors for suicide attempt in pregnancy and the post-partum period in women with serious mental illnesses. J Psychiatr Res. 2017;84:284-291. 